Pacific Journal of Mathematics

SMALL DOWER SPACES

M Albert RODERICK WeISS 


\title{
SMALL DOWKER SPACES
}

\author{
W. WEISS
}

\begin{abstract}
We construct a normal, locally compact, first countable, separable, real compact topological space which is not countabley paracompact. This construction is performed under the (relatively consistent) set-theoretic hypotheses: Martin's Axiom plus $\diamond_{\mathrm{c}}(E)$.
\end{abstract}

Years ago, C. Dowker proved the now well-known theorem that for any Hausdorff topological space $X$, topological product of $X$ with the closed unit interval is normal iff $X$ is both normal and countably paracompact [3]. Since then researchers have called Hausdorff spaces which are normal but not countably paracompact Dowker spaces.

Dowker spaces seem to be extremely rare and difficult to construct. In fact, in the literature there is only one which is constructed using just the usual ZFC axioms of set theory [8]. It was discovered by M. E. Rudin who then asked [10] if there were any "small" Dowker spaces, i.e., ones which are, for example, first countable, separable, realcompact, or of small cardinality.

Examples have been constructed, using extra set-theoretic axioms. M. E. Rudin [9] used the existence of a Suslin line to obtain a Dowker space which is hereditarily separable and first countable. In [5] I. Juhász, K. Kunen and M. E. Rudin construct, using $\mathrm{CH}$, a first countable, hereditarily separable, realcompact Dowker space and claim that with the stronger assumption $\diamond$ they could construct one which is locally compact as well.

It was unknown if Martin's axiom plus not $\mathrm{CH}$ allowed the construction of small Dowker spaces since Martin's axiom plus not $\mathrm{CH}$ often implies topological results contrary to $\mathrm{CH}$ or $\diamond$. In this paper we construct a Dowker space using axioms of set theory consistent with Martin's axiom. This Dowker space is locally compact, first countable, separable and realcompact. By the results of [11] we cannot hope to prove that this Dowker space is hereditarily separable.

Recently, M. Bell has constructed a first countable realcompact Dowker space, assuming Martin's axiom. In fact, only a weakened form of Martin's axiom, called MA ( $\sigma$-centered) is used. We use it repeatedly in our construction.

MA ( $\sigma$-centered). If $\boldsymbol{P}$ is a partial order such that $\boldsymbol{P}=\mathbf{U}_{n \in \omega} \boldsymbol{P}_{n}$ where each $\boldsymbol{P}_{n}$ is centered and $\kappa<2^{\boldsymbol{N}_{0}}$ and $\left\{\boldsymbol{D}_{\alpha}: \alpha<\kappa\right\}$ is a collection of dense subsets of $\boldsymbol{P}$, then $\boldsymbol{P}$ contains a $\left\{\boldsymbol{D}_{\alpha}: \alpha<\kappa\right\}$-generic subset. Recall that a subset $S$ of a partial order $\boldsymbol{P}$ is centered iff each 
finite subset of $S$ has a lower bound. A subset $D$ of $\boldsymbol{P}$ is called dense iff for each $p \in \boldsymbol{P}$ there is some element of $D$ less than or equal to $p$. If $\left\{D_{\alpha}: \alpha<\kappa\right\}$ is a collection of dense of subset of $\boldsymbol{P}$, a subset $G$ of $\boldsymbol{P}$ is called $\left\{D_{\alpha}: \alpha<\kappa\right\}$-generic iff $D_{\alpha} \cap G \neq \varnothing$ for each $\alpha<\kappa$.

Another useful axiom is variation of $\diamond$, called $\nabla_{\omega_{2}}(E)$. We let $E=\left\{\alpha \in \omega_{2}: \operatorname{cf}(\alpha)=\omega\right\}$.

$\diamond_{\omega_{2}}(E)$. There is a sequence $\left\{X_{\alpha}: \alpha \in E\right\}$ such that each $X_{\alpha} \subseteq \alpha$ and for any $X \subseteq \omega_{2}\{\alpha \in E: X=X \cap \alpha\}$ is stationary in $\omega_{2}$.

Proposition 1. If ZFC is consistent, so is ZFC plus MA ( $\sigma$ centered) plus $2^{\aleph_{0}}=\boldsymbol{\aleph}_{2}$ plus $\diamond_{\omega_{2}}(E)$.

Proof. This seems to be folklore. The model needed can be the "usual" model of Martin's axiom plus $2^{\aleph_{0}}=\boldsymbol{\aleph}_{2}$ where the iterated forcing is done over a ground model of $V=L$. A modification of the proof of Theorem 7 of Chapter 18 in [2] shows that this model is a model of $\diamond_{\omega_{2}}(E)$.

The role of $\boldsymbol{W}_{2}$ in the collection of axioms in Proposition 1 might be played, in this article, by any regular uncountable cardinal, but for concreteness we choose to assume $2^{\aleph_{0}}=\boldsymbol{\aleph}_{2}$. These assumptions are used by Hajnal and Juhász for a topological theorem of a completely different nature in [4].

Proposition 2. $\diamond_{\omega_{2}}(E)$ implies there exists a sequence of ordered pairs $\left\{\left\langle S_{\alpha}, T_{\alpha}\right\rangle: \alpha \in E\right\}$ such that for all $\alpha \in E S_{\alpha} \leqq \alpha$ and $T_{\alpha} \leqq \alpha$ and for any two subsets $S$ and $T$ of $\omega_{2},\left\{\alpha \in E\right.$ : both $S_{\alpha}=S \cap \alpha$ and $T_{\alpha}=$ $T \cap \alpha\}$ is stationary in $\omega_{2}$.

Proof. This too is folklore. Briefly, let $f: \omega_{2} \rightarrow \omega_{2} \times \omega_{2}$ be any bijection. Let $S_{\alpha}=$ first coordinates of elements of $\left.f^{\prime \prime} X_{\alpha}\right\}$ and $T_{\alpha}=$ \{second coordinates of elements of $f^{\prime \prime} X_{\alpha}$. Then for any two subsets $S$ and $T$ of $\omega_{2}\left\{\alpha \in E: X_{\alpha}=f^{-1}(S \times T) \cap \alpha\right\} \cap\left\{\alpha \in \omega_{2}: f^{\prime \prime} \alpha=\alpha \times \alpha\right\}$ is contained in $\left\{\alpha \in E: S_{\alpha} \times T_{\alpha}=(S \cap \alpha) \times(T \cap \alpha)\right\}$ which must therefore be stationary.

We denote the real numbers by $\boldsymbol{R}$ and the rationals by $\boldsymbol{Q}$. In [5], Lusin sets are used to construct a Dowker space. Here, we use:

Proposition 3. MA ( $\sigma$-centered) plus $2^{\aleph_{0}}=\aleph_{2}$ implies there is an $\boldsymbol{L} \subseteq \boldsymbol{R}$ such that

(i) $\boldsymbol{L} \cap \boldsymbol{Q}=\varnothing$, 
(ii) for any open $U \subseteq \boldsymbol{R},|\boldsymbol{L} \cap U|=\boldsymbol{\aleph}_{2}$,

(iii) for any nowhere dense $N \subseteq \boldsymbol{R}|\boldsymbol{L} \cap N|<\boldsymbol{\aleph}_{2}$.

Proof. Folklore again. Note that the hypotheses imply that $\boldsymbol{R}$ is not the union of $\leqq \boldsymbol{\aleph}_{1}$ nowhere dense sets and then make a simple modification to the usal inductive construction of a Lusin set.

We now do the promised construction. We will eventually define a topology $\tau$ on a subset $X$ of $\boldsymbol{L} \cup \boldsymbol{Q}$, where $\boldsymbol{L}$ is as given in Proposition 3. Fix $\leqq$ as a well ordering of $L$. Let $\left\{A_{\alpha}: \alpha \in \omega_{2} \backslash E\right\}$ enumerate $\left\{A \subseteq \boldsymbol{L}:|A|<\boldsymbol{\aleph}_{2}\right\}$ with each $A$ being repeated $\boldsymbol{\aleph}_{2}$ times. In addition, let $\left\{B_{\alpha}: \alpha \in \omega_{2} \backslash E\right\}$ enumerate all countable sequences of subsets of $\boldsymbol{Q}$. Finally, let $\left\{\left\langle S_{\alpha}, T_{\alpha}\right\rangle: \alpha \in E\right\}$ be as in Proposition 2.

From now on we shall assume ZFC plus MA ( $\sigma$-centered) plus $2^{\aleph_{0}}=\aleph_{2}$ plus $\diamond_{\omega_{2}}(E)$.

We shall define the topology $\tau$ on $X \subseteq L \cup \boldsymbol{Q}$ by recursively defining a sequence of points $\left\{x_{\alpha}: \alpha \in \omega_{2}\right\} \cong \boldsymbol{L}$ and topologies $\tau_{\beta}$ on $X_{\beta}=\left\{x_{\alpha}: \alpha \in \beta\right\} \cup \boldsymbol{Q}$, for all $\beta \in \omega_{2}$. Simultaneously we define a collection $\left\{C_{\alpha}: \alpha \in \omega_{2} \backslash E\right\}$ of subsets of $\boldsymbol{L} \cup \boldsymbol{Q}$ and a function $\rho: X \rightarrow \omega$ recursively.

The inductive hypothesis is that for all $\alpha<\beta$

(i ) $\tau_{\alpha}=\tau_{\beta} \cap \mathscr{P}\left(X_{\alpha}\right)$,

(ii ) $\tau_{\beta}$ refines the Euclidean topology on $X_{\beta}$,

(iii) $\boldsymbol{Q}$ is dense in $\left\langle X_{\beta}, \tau_{\beta}\right\rangle$,

(iv) $\tau_{\beta}$ is a locally compact, locally countable topology,

(v) each $C_{\alpha}$ is a clopen subset of $\left\langle X_{\beta}, \tau_{\beta}\right\rangle$,

(vi) $\rho\left(x_{\alpha}\right)=n$ implies there exists $U \in \tau_{\alpha+1}$ such that for all $y \in U$ $\rho(y) \leqq n$.

(vii) for all finite $F \subseteq \beta, \boldsymbol{Q} \backslash \bigcup_{\alpha \in F} C_{\alpha}$ is dense in the Euclidean topology on $\boldsymbol{R}$.

We shall make the construction so that each subset of $\boldsymbol{L}$ of cardinality $<\boldsymbol{K}_{2}$ is contained in some $C_{\alpha}$.

We begin the construction by letting $X_{0}=\boldsymbol{Q}$ and $\tau_{0}$ the discrete topology on $\boldsymbol{Q}$.

At limit ordinals $\lambda$, including $\lambda=\omega_{2}$, we let $\tau_{\lambda}$ be the topology on $X_{\lambda}=\bigcup\left\{X_{\beta}: \beta \in \lambda\right\}$ generated by $\bigcup\left\{\tau_{\beta}: \beta \in \lambda\right\}$. The inductive hypothesis is easily seen to be satisfied. All the action takes place at successor stages and there are two cases to consider: first, when $\beta=\alpha+1$ for some $\alpha \in E$ and second, when $\beta=\alpha+1$ for some $\alpha \notin E$.

At stage $\alpha+1$ when $\alpha \in E$ we consider $\left\langle S_{\alpha}, T_{\alpha}\right\rangle$ and an increasing sequence $\left\{\alpha_{n}: n \in \omega\right\}$ converging to $\alpha$.

Case (a). There is $x \in L \backslash X_{\alpha}$, there is a sequence $\left\{s_{n}: n \in \omega\right\} \subseteq S_{\alpha}$ 
and a sequence $\left\{t_{n}: n \in \omega\right\} \subseteq T_{\alpha}$ such that:

(i) each $s_{n} \in X_{\alpha} \backslash X_{\alpha_{n}}$ and each $t_{n} \in X_{\alpha} \backslash X_{\alpha_{n}}$,

(ii) there exists $l \in \omega$ such that $\left\{\rho\left(s_{n}\right): n \in \omega\right\} \subseteq l$ and $\left\{\rho\left(t_{n}\right)\right.$ : $n \in \omega\} \subseteq l$,

(iii) in the Euclidean topology on $\boldsymbol{R}\left\{S_{n}\right\} \rightarrow x$ and $\left\{t_{n}\right\} \rightarrow x$.

In this case, let $x_{\alpha}=x$. We need a lemma.

LEMMA 1. There exist open $U_{n} \in \tau_{\alpha}$ containing $s_{n}$ such that for all $\xi<\alpha\left\{n \in \omega: C_{\xi} \cap U_{n} \neq \varnothing\right\}$ is finite.

Proof. Since $\left\langle X_{\alpha}, \tau_{\alpha}\right\rangle$ is first countable, let $V(n, m)$ be the $m$ th basic open neighborhood of $s_{n}$.

Let $\boldsymbol{P}$ be the set of all pairs $\langle f, g\rangle$ such that

(i) $f$ is a finite partial function from $\alpha$ into $\omega$,

(ii) $g$ is a finite partial function from $\omega$ into $\omega$, and

(iii) for all $\xi \in \operatorname{dom} f$, for all $n \in \operatorname{dom} g, n>f(\xi)$ implies

$$
s_{n} \in C_{\xi} \quad \text { or } \quad V(n, g(n)) \cap C_{\xi}=\varnothing ，
$$

with the partial ordering of function extension in both coordinates.

Let $\boldsymbol{D}_{n}^{1}=\{\langle f, g\rangle: n \in \operatorname{dom} g\}$. Since each $C_{\xi}$ is closed in $\left\langle X_{\alpha}, \tau_{\alpha}\right\rangle$, each $\boldsymbol{D}_{n}^{1}$ is dense in $\boldsymbol{P}$.

Let $\boldsymbol{D}_{\xi}^{2}=\{\langle f, g\rangle: \xi \in \operatorname{dom} f\}$. Clearly each $\boldsymbol{D}_{\xi}^{2}$ is dense in $\boldsymbol{P}$.

Now, if $\left\langle f_{2}, g\right\rangle$ and $\left\langle f_{2}, g\right\rangle$ are elements of $\boldsymbol{P}$ and $f_{1}$ and $f_{2}$ are compatible as functions, then $\left\langle f_{1} \cup f_{2}, g\right\rangle$ is in $\boldsymbol{P}$. Hence for each $\widetilde{g}$, $\{\langle f, g\rangle: g=\widetilde{g}\}$ is isomorphic to the partial order of finite partial functions from $\alpha$ into $\omega$ ordered by set containment, and so $\boldsymbol{P}$ is $\sigma$-centered.

By MA ( $\sigma$-centered), there is a $\boldsymbol{G} \subseteq \boldsymbol{P}$ generic for all $\boldsymbol{D}_{n}^{1}$ and $\boldsymbol{D}_{\xi}^{2}$. Let $F=\bigcup\{f$ : for some $g,\langle f, g\rangle \in G\}$ and $G=\bigcup\{g$ : for some $f$, $\langle f, g\rangle \in \boldsymbol{G}\}$.

Let $U_{n}=V(n, G(n))$. For each $\xi \in \alpha$ there is some $n \in \omega$ such that $C_{\xi} \subseteq X_{\alpha_{n \xi}}$ If $n>\max \left\{n_{\xi}, F(\xi)\right\}$, then $U_{n} \cap C_{\xi}=\varnothing$.

Returning to the construction, let $\left\{O_{s}(n): n \in \omega\right\}$ refine $\left\{U_{n}: n \in \omega\right\}$ of Lemma 1 such that

(i ) $s_{n} \in O_{s}(n) \subseteq U_{n}, \rho^{\prime \prime} O_{s}(n) \subseteq l$,

(ii) $O_{s}(n)$ is countable, compact and open in $\left\langle X_{\alpha}, \tau_{\alpha}\right\rangle$,

(iii) the Euclidean diameter diam $O_{s}(n)<1 / n$ for each $n$.

Similarly obtain $\left\{O_{t}(n): n \in \omega\right\}$ with each $t_{n} \in O_{t}(n)$. Let $V_{m}=$ $\left\{x_{\alpha}\right\} \cup \bigcup\left\{O_{s}(n): n \geqq m\right\} \cup \bigcup\left\{O_{t}(n): n \geqq m\right\}$. Let $\tau_{\alpha} \cup\left\{V_{m}: m \in \omega\right\}$ generate $\tau_{\alpha+1}$. Let $\rho\left(x_{\alpha}\right)=l$. A routine check shows that the inductive hypothesis is still satisfied.

Case (b). If the conditions for Case (a) are not satisfied, we 
waste time with the help of this lemma.

LEMma 2. There is $a \mathbf{Q}^{*} \subseteq \boldsymbol{Q}$ which is dense in the Euclidean topology and for all $\xi<\alpha,\left(\boldsymbol{Q}^{*} \cap C_{\xi}\right)$ is finite.

Proof. Enumerate $\boldsymbol{Q}$ as $\left\{q_{n}: n \in \omega\right\}$. Let $\boldsymbol{P}$ be the set of all ordered pairs $\langle M, f\rangle$ such that

(i) $M$ is a finite subset of $\boldsymbol{Q}$,

(ii) $f$ is a finite partial function from $\alpha$ into $\omega$,

(iii) if $\xi \in \operatorname{dom} f$ then $C_{\xi} \cap M \subseteq\left\{q_{n}: n<f(\xi)\right\}$ with the partial order given by extension of each coordinate.

Let $\boldsymbol{D}_{\xi}=\{\langle M, f\rangle: \xi \in \operatorname{dom} f\}$. Clearly $\boldsymbol{D}_{\xi}$ is dense in $\boldsymbol{P}$ for each $\xi<\alpha$.

Let, for each pair of rationals $p, r$ with $p<r, D_{p, r}=\{\langle M, f\rangle$ : $M \cap(p, r) \neq \varnothing\}$, where $(p, r)$ is the open interval. Condition (vii) of the inductive hypothesis ensures that each $\boldsymbol{D}_{p, r}$ is dense.

For each $\tilde{M},\{\langle M, f\rangle: M=\tilde{M}\}$ is isomorphic to the partial order of finite partial functions from $\alpha$ into $\omega$ ordered by set containment, and hence $\boldsymbol{P}$ is $\sigma$-centered.

Hence by MA ( $\boldsymbol{\sigma}$-centered) there is a $\boldsymbol{G} \cong \boldsymbol{P}$ which is generic with respect to the above-mentioned dense sets. Let $\boldsymbol{Q}^{*}=\mathbf{U}\{M$ : there is some $f$ such that $\langle M, f\rangle \in G\}$, and let $F=\bigcup\{f$ : there is some $M$ such that $\langle M, f\rangle \in G\}$. Then $Q^{*}$ is dense and for each $\xi<\alpha,\left(\boldsymbol{Q}^{*} \cap C_{\xi}\right) \leqq\left\{q_{n}: n<F(\xi)\right\}$.

We can now pick any $x \in \boldsymbol{L} \backslash X_{\alpha}$ and find $\left\{p_{n}: n \in \omega\right\} \subseteq \boldsymbol{Q}^{*}$ from Lemma 2 such that $\left\{p_{n}\right\} \rightarrow x$ in the Euclidean topology. Let $x_{\alpha}=x$ and let $V_{m}=\left\{x_{\alpha}\right\} \cup\left\{p_{n}: n \geqq m\right\}$. Let $\tau_{\alpha+1}$ be generated by $\tau_{\alpha} \cup\left\{V_{m}: m \in \omega\right\}$ and let $\rho\left(x_{\alpha}\right)=0$ to satisfy the inductive hypothesis in this case.

We can now proceed to stage $\alpha+1$ where $\alpha \notin E$. We must do two things: define $x_{\alpha}$ and $\tau_{\alpha+1}$ and define $C_{\alpha}$.

Embarking on the first task, let's consider $B_{\alpha}=\left\langle B_{\alpha}^{0}, B_{\alpha}^{1}, B_{\alpha}^{2}, \cdots\right\rangle$. Using Lemma 2 obtain $\boldsymbol{Q}^{*} \subseteq \boldsymbol{Q}$, dense, such that $C_{\xi} \cap \boldsymbol{Q}^{*}$ is finite for each $\xi<\alpha$. There are now two cases to consider.

Case (a). Each $x \in L \backslash X_{\alpha}$ is in the Euclidean closure of only finitely many of the sets $B_{\alpha}^{j} \cap Q^{*}, j \in \omega$. In this case let $x_{\alpha}$ be any element of $\boldsymbol{L} \backslash X_{\alpha}$. Pick $\left\{p_{n}: n \in \omega\right\} \cong \boldsymbol{Q}^{*}$ such that $\left\{p_{n}\right\} \rightarrow x_{\alpha}$ in the Euclidean topology. Let $\tau_{\alpha} \cup\left\{\left\{x_{\alpha}\right\} \cup\left\{p_{n}: n \supseteqq m\right\}: m \in \omega\right\}$ generate $\tau_{\alpha+1}$. Let $\rho\left(x_{\alpha}\right)=1+\max \left\{j: x_{\alpha}\right.$ is in the Euclidean closure of $\left.B_{\alpha}^{j} \cap \boldsymbol{Q}^{*}\right\}$. Note that the inductive hypothesis is satisfied and that for all $j \supseteqq \rho\left(x_{\alpha}\right), x_{\alpha} \notin \tau_{\alpha+1}$-closure of $B_{\alpha}^{j}$.

Case (b). There is some $x \in \boldsymbol{L} \backslash X_{\beta}$ such that $x$ is in the Euclidean 
closure of infinitely many of the sets $B_{\alpha}^{j} \cap Q^{*}$. In this case pick $x_{\alpha}$ to be some such $x$. Pick an infinite $J \subseteq \omega$ such that for each $j \in J, x_{\alpha}$ is in the Euclidean closure of $B_{\alpha}^{j} \cap Q^{*}$. For each $j \in J$ pick $\left\{p_{n}^{j}: n \in \omega\right\} \subseteq$ $B_{\alpha}^{j} \cap Q^{*}$ such that $\left\{p_{n}^{j}\right\} \rightarrow x_{\alpha}$ in the Euclidean topology. Let $\left\{r_{k}: k \in \omega\right\}$ be a subsequence of $\left\{p_{n}^{j}: n \in \omega, j \in J\right\}$ such that for each $j \in J$ each tail of $\left\{r_{k}\right\}$ contains an infinite subset of $\left\{p_{n}^{j}: n \in \omega\right\}$ and such that $\left\{r_{k}\right\} \rightarrow x_{\alpha}$ in the Euclidean topology. Let $\tau_{\alpha} \cup\left\{\left\{x_{\alpha}\right\} \cup\left\{r_{k}: k \geqq m\right\}: m \in \omega\right\}$ generate $\tau_{\alpha+1}$. Let $\rho\left(x_{\alpha}\right)=0$. Note that the inductive hypothesis is satisfied and that $x_{\alpha}$ is in the $\tau_{\alpha+1}$-closure of infinitely many $E_{a}^{j}$.

We now proceed to define $C_{\alpha}$. Consider $A_{\alpha}$. If $A_{\alpha} \nsubseteq X_{\alpha+1}$, let $C_{\alpha}=\varnothing$. If $A_{\alpha} \leqq X_{\alpha+1}$, then $C_{\alpha}$ is given by the following lemma.

Lemma 3. Suppose $A_{\alpha} \subseteq\left(X_{\alpha+1} \cap L\right)$ and $\left\{C_{\xi}: \xi \in \alpha \backslash E\right\}$ is a collection of clopen subsets of $X_{\alpha+1}$ such that for each finite $F \cong(\alpha \backslash E)$, $\left(Q \backslash \bigcup\left\{C_{\xi}: \xi \in F\right\}\right)$ is dense in the Euclidean topology. Then there exists a clopen $C_{\alpha} \leqq X_{\alpha+1}$ such that $A_{\alpha} \subseteq C_{\alpha}$ and for each finite $F \subseteq((\alpha+$ 1) $\backslash E),\left(Q \backslash \bigcup\left\{C_{\xi}: \xi \in F\right\}\right)$ is dense in the Euclidean topology.

Proof. From Lemma 1 obtain $\boldsymbol{Q}^{*}$ such that for each $\xi \in(\alpha \backslash E)$, $\left(\boldsymbol{Q}^{*} \cap C_{\xi}\right)$ finite, and $\boldsymbol{Q}^{*}$ is dense in the Euclidean topology. It now suffices to construct a clopen $C_{\alpha}$ such that $A_{\alpha} \subseteq C_{\alpha} \subseteq X_{\alpha+1}$ and $\left(Q^{*} \backslash C_{\alpha}\right)$ is dense.

To this end let $\boldsymbol{P}$ be the set of all triples $\langle a, b, c\rangle$ such that:

(i) $a$ and $b$ are clopen, compact subsets of $X_{\alpha+1}$,

(ii) $c$ is a finite subset of $\boldsymbol{Q}^{*}$,

(iii) $(a \cup A) \cap b=\varnothing$,

(iv) $a \cap c=\varnothing$

with the partial order of set containment in each coordinate.

$\{\langle a, b, c\rangle: x \in a \cup b\}$ is dense for each $x \in X_{\alpha+1}$. For each interval $(p, q)$ with rational endpoints, $\langle a, b, c\rangle:(p, q) \cap c \neq \varnothing\}$ is dense.

For each finite union $I$ of interval with rational endpoints and each finite $\widetilde{c} \subseteq Q^{*},\{\langle a, b, c\rangle: a \leqq I,(b \cap I)=\varnothing$ and $c=\widetilde{c}\}$ is centered; hence $\boldsymbol{P}$ is $\sigma$-centered.

Let $\boldsymbol{G} \subseteq \boldsymbol{P}$ be generic with respect to the above dense sets. Let $C_{\alpha}=\bigcup\{a$ : for some $b$ and $c,\langle a, b, c\rangle \in G\}$. It is straightforward to show that $C_{\alpha}$ is clopen, $A_{\alpha} \subseteq C_{\alpha}$ and $\left(Q^{*} \mid C_{\alpha}\right)$ is dense.

This completes the construction. We let $X=X_{\omega_{2}}$ and $\tau=\tau_{\omega_{2}}$. Clearly $\tau$ is a locally compact, $T_{2}$, first countable, separable and submetrizable topology on $X$. Since $\langle X, \tau\rangle$ is first countable and submetrizable, it is real-compact [5] also. It remains to show that $\langle X, \tau\rangle$ is a Dowker space.

We show that $\langle X, \tau\rangle$ is not countably compact by showing that the open cover $\left\{O_{j}: j \in \omega\right\}$ has no precise locally finite refinement, 
where $O_{j}=\{x \in X: \rho(x) \leqq j\}$. Due to (vi) of the inductive hypothesis $\left\{O_{j}: j \in \omega\right\}$ is an open cover. In order to achieve a contradiction assume $\left\{W_{j}: j \in \omega\right\}$ is a locally finite precise refinement. For some $\alpha \in\left(\omega_{2} \backslash E\right),\left\langle\left(W_{0} \cap \boldsymbol{Q}\right),\left(W_{1} \cap \boldsymbol{Q}\right),\left(W_{2} \cap \boldsymbol{Q}\right), \cdots\right\rangle=B_{\alpha}$ and we look at what happened at stage $\alpha+1$. Under Case (b) $x_{\alpha}$ witnesses that $\left\{W_{j}: j \in \omega\right\}$ is not locally finite. Under Case (a) $x_{\alpha}$ witnesses that $\left\{W_{j}: j \in \omega\right\}$ is not a cover, since if $j<\rho\left(x_{\kappa}\right)$ then $x_{\kappa} \notin O_{j}$ and if $j \geqq$ $\rho\left(x_{k}\right)$ then $x_{\alpha} \notin \overline{W_{j} \cap \boldsymbol{Q}} \supseteq W_{j}$.

In order to show that $\langle X, \tau\rangle$ is normal, we need a short lemma.

Lemma 4. If $H$ and $K$ are closed disjoint subsets of $X$, one of which has cardinality $<\boldsymbol{\aleph}_{2}$, then there are disjoint open sets separating $H$ and $K$.

Proof. Assume $|H|<\aleph_{2}$ so that there is some clopen $C \subseteq X$ such that $|C|<\boldsymbol{\aleph}_{2}$ and $H \subseteq C$. Let $K^{*}=K \cap C$. By MA ( $\sigma$-centered), since $X$ is locally compact and submetrizable there exist disjoint open $U$ and $V$ such that $H \subseteq U$ and $K^{*} \subseteq V[1,6]$. Then $H \subseteq U \cap C$ and $K \subseteq U \cup(X \backslash C)$ separating $H$ and $K$.

Now let $H$ and $K$ be arbitrary closed disjoint subsets of $X$. Without loss of generality we assume $H \cup K \subseteq \boldsymbol{L}$ and we find disjoint open sets separatirg $H$ and $K$. Let $H_{i}=\{x \in H: \rho(x) \leqq i\}$ and $K_{i}=$ $\{x \in K: \rho(x) \leqq i\}$. Define $U_{i}^{I I}$ to the compliment in $\boldsymbol{R}$ of the Euclidean closure of $\bigcup\left\{V: V\right.$ is Euclidean open and $\left.\left|V \cap H_{i}\right|<\boldsymbol{\aleph}_{2}\right\}$. Note that for each nonempty Euclidean open $V \subseteq U_{i}^{H}\left|V \cap H_{i}\right|=\boldsymbol{\aleph}_{2}$. Similarly define $U_{i}^{K}$ for each $i \in \omega$.

Lemma 5. With $U_{i}^{\prime \prime}$ and $U_{i}^{K}$ defined as above for each $i \in()$ we have $\bigcup_{i \in \omega} U_{i}^{K} \cap \bigcup_{i \in \omega} U_{i}^{I I}=\varnothing$.

Proof. Suppose $U_{i}^{I I} \cap U_{j}^{K} \neq \varnothing$. Then there is some interval $I \leqq U_{i}^{I I} \cap U_{j}^{K}$. Let $\hat{H}=H_{i} \cap I$ and $\hat{K}=K_{j} \cap I$. Let $Y_{I I}=\left\{\alpha \in \omega_{2}\right.$ : for all $\beta<\alpha \hat{H} \cap[\beta, \alpha)$ is dense in $I\}$ and $Y_{K}=\left\{\alpha \in \omega_{2}\right.$ : for all $\beta<\alpha \hat{K} \subset[\beta, \alpha)$ is dense in $I\} . Y_{I I}$ and $Y_{K}$ are both cub subsets of $\omega_{2}$. So there exists, by $\diamond_{\omega_{2}}(E)$, some $\alpha \in E \cap Y_{H} \cap Y_{K}$ such that $\hat{H} \cap \alpha=S_{\alpha}$ and $\hat{K} \cap \alpha=T_{\alpha}$.

Let's see what happened at stage $\alpha+1$. We had $\left\{\alpha_{n}: n \in \omega\right\}$ increasing up to $\alpha$. Since $\alpha \in Y_{I I} \cap Y_{K}$ we can find $x \in \boldsymbol{L} \backslash X_{\alpha}$ and sequences $\left\{s_{n}: n \in \omega\right\}$ and $\left\{t_{n}: n \in \omega\right\}$ such that the conditions for Case (a) hold. Hence $x_{\alpha} \in \bar{S}_{\alpha} \cap \bar{T}_{\alpha} \subseteq \bar{H} \cap \bar{K}$ which is a contradiction.

Now, since $\boldsymbol{L}$ has the properties from Proposition 3, both $H \backslash \bigcup_{i \in \omega} U_{i}^{H}$ and $K \backslash \bigcup_{i \in \omega} U_{k}^{K}$ have cardinality less than $\boldsymbol{\aleph}_{2}$. Hence by Lemma 4 there are open $V_{I I}$ and $V_{K}$ such that: 
and

$$
\left(H \backslash \bigcup_{i \in \omega} U_{i}^{I I}\right) \leqq V_{H} \subseteq \bar{V}_{H} \leqq(X \backslash K)
$$

Hence

$$
\left(K \backslash \bigcup_{\imath \in \omega} U_{i}^{K}\right) \subseteq V_{K} \subseteq \bar{V}_{K} \subseteq(X \backslash H)
$$

$$
H \leqq\left(V_{H} \backslash \bar{V}_{K}\right) \cup \bigcup_{i \in \omega} U_{i}^{H}
$$

and

$$
K \subseteq\left(V_{K} \backslash \bar{V}_{H}\right) \cup \bigcup_{i \in \omega} U_{i}^{K}
$$

which separates $H$ and $K$. The proof is complete.

We could do the above constructions and proofs with $\aleph_{2}$ replaced everywhere by $\boldsymbol{\aleph}_{1}$ thus reducing the set theoretic assumptions to ZFC plus $\diamond$. A Dowker space with properties similar to $X$ was claimed in [5] to follow from $\diamond$. The additional property of hereditary separability mentioned in [5] can be obtained by weaving in the idea of constructing hereditarily separable spaces from $\diamond$ in [7] to the above construction.

The space $\langle X, \tau\rangle$ above is normal and each bounded or closed unbounded (referring to the indices) subspace is also normal. However, there is no reason to believe that $\langle X, \tau\rangle$ is hereditarily normal.

\section{REFERENCES}

1. K. Alster and T. Przymusinski, Normality and Martin's axiom, Fund. Math., 41 (1976), 123-131.

2. K. J. Devlin, Aspects of Constructibility, Lecture Notes in Math., Vol. 354, SpringerVerlag, Berlin and New York, 1973.

3. C. H. Dowker, On countably paracompact spaces, Canad. J. Math., 3 (1951), 219-224.

4. A. Hajnal and I. Juhász, Weakly separated subspaces and networks, preprint.

5. I. Juhász, K. Kunen and M. E. Rudin, Two more hereditarily separable non-Lindelöf spaces, Canad. J. Math., 28 (1976), 998-1005.

6. I. Juhász and W. Weiss, Martin's axiom and normality, General Topology and Appl., 9 (1978), 263-274.

7. A. Ostaszewski, On countably compact, perfectly normal spaces, J. London Math. Soc., 14 (1976), 505-516.

8. M. E. Rudin, A normal space for which $X \times I$ is not normal, Fund. Math., 78 (19711972), 179-168.

9. — A separable Dowker space, Symposia Mathematica, Instituto Nazionale di Alta Mathematica, 1973.

10. Lectures in Set-theoretic Topology, Conference Board of the Mathematical Sciences Regional Conference Series in Mathematics No., 23 (1974); Amer. Math. Soc., Providence, R.I.

11. Z. Szentmiklóssy, $S$ and L spaces under MA, to appear.

Received October 9, 1979 and in revised form December 14, 1979. Research partially supported by NSTRC grant no. A3185.

UnIVERSITY OF TORONTO

Toronto M5S 1A1, Canada 


\section{PACIFIC JOURNAL OF MATHEMATICS}

\section{EDITORS}

DONALD BABBITT (Managing Editor)

University of California

Los Angeles, CA 90024

Hugo RossI

University of Utah

Salt Lake City, UT 84112

C. C. MOORE and ANDREW OGG

University of California

Berkeley, CA 94720
J. DugundjI

Department of Mathematics

University of Southern California

Los Angeles, CA 90007

R. FINN and J. MILGRAM

Stanford University

Stanford, CA 94305

\section{ASSOCIATE EDITORS}
R. ARENS
E. F. BECKENBACH
B. H. NeUmanN
F. WOLF
K. YosHIDA

\section{SUPPORTING INSTITUTIONS}

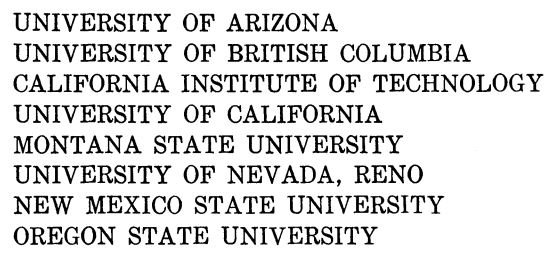

UNIVERSITY OF ARIZONA

UNIVERSITY OF BRITISH COLUMBIA CALIFORNIA INSTITUTE OF TECHNOLOGY

UNIVERSITY OF CALIFORNIA

MONTANA STATE UNIVERSITY

UNIVERSITY OF NEVADA, RENO

NEW MEXICO STATE UNIVERSITY OREGON STATE UNIVERSITY

\author{
UNIVERSITY OF OREGON \\ UNIVERSITY OF SOUTHERN CALIFORNIA \\ STANFORD UNIVERSITY \\ UNIVERSITY OF HAWAII \\ UNIVERSITY OF TOKYO \\ UNIVERSITY OF UTAH \\ WASHINGTON STATE UNIVERSITY \\ UNIVERSITY OF WASHINGTON
}

The Supporting Institutions listed above contribute to the cost of publication of this Journal, but they are not owners or publishers and have no responsibility for its content or policies.

Mathematical papers intended for publication in the Pacific Journal of Mathematics should be in typed form or offset-reproduced, (not dittoed), double spaced with large margins. Please do not use built up fractions in the text of the manuscript. However, you may use them in the displayed equations. Underline Greek letters in red, German in green, and script in blue. The first paragraph or two must be capable of being used separately as a synopsis of the entire paper. Please propose a heading for the odd numbered pages of less than 35 characters. Manuscripts, in triplicate, may be sent to any one of the editors. Please classify according to the scheme of Math. Reviews, Index to Vol. 39. Supply name and address of author to whom proofs should be sent. All other communications should be addressed to the managing editor, or Elaine Barth, University of California, Los Angeles, California, 90024.

50 reprints to each author are provided free for each article, only if page charges have been substantially paid. Additional copies may be obtained at cost in multiples of 50 .

The Pacific Journal of Mathematics is issued monthly as of January 1966. Regular subscription rate: $\$ 102.00$ a year (6 Vols., 12 issues). Special rate: $\$ 51.00$ a year to individual members of supporting institutions.

Subscriptions, orders for numbers issued in the last three calendar years, and changes of address shoud be sent to Pacific Journal of Mathematics, P.O. Box 969, Carmel Valley, CA 93924, U.S.A. Old back numbers obtainable from Kraus Per!odicals Co., Route 100, Millwood, NY 10546.

\section{PUBLISHED BY PACIFIC JOURNAL OF MATHEMATICS, A NON-PROFIT CORPORATION}

Printed at Kokusai Bunken Insatsusha (International Academic Printing Co., Ltd.). 8-8, 3-chome, Takadanobaba, Shinjuku-ku, Tokyo 160, Japan.

Copyright (C) 1981 by Pacific Jounal of Mathematics Manufactured and first issued in Japan 


\section{Pacific Journal of Mathematics}

Vol. 94, No. $2 \quad$ June, 1981

Thomas E. Armstrong and William David Sudderth, Nearly strategic

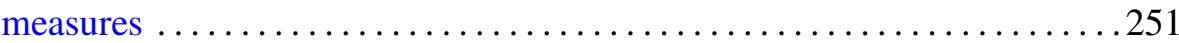

John J. Buoni, Artatrana Dash and Bhushan L. Wadhwa, Joint Browder

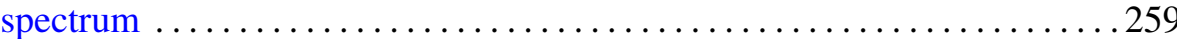

Jack Paul Diamond, Hypergeometric series with a $p$-adic variable . .......265

Raymond Frank Dickman, Jack Ray Porter and Leonard Rubin,

Completely regular absolutes and projective objects ............ 277

James Kenneth Finch, On the local spectrum and the adjoint ........... 297

Benno Fuchssteiner, An abstract disintegration theorem ............ 303

Leon Gerber, The volume cut off a simplex by a half-space $\ldots \ldots \ldots \ldots 311$

Irving Leonard Glicksberg, An application of Wermer's subharmonicity

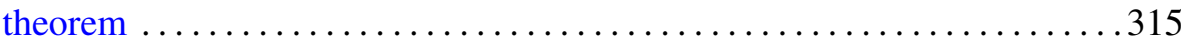

William Goldman, Two examples of affine manifolds ............... 327

Yukio Hirashita, On the Weierstrass points on open Riemann surfaces .....331

Darrell Conley Kent, A note on regular Cauchy spaces ............. 333

Abel Klein and Lawrence J. Landau, Periodic Gaussian

Osterwalder-Schrader positive processes and the two-sided Markov

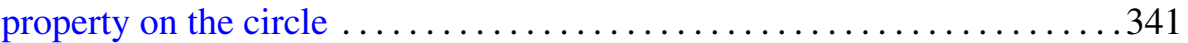

Brenda MacGibbon, $\mathscr{K}$-Borelian embeddings and images of Hausdorff

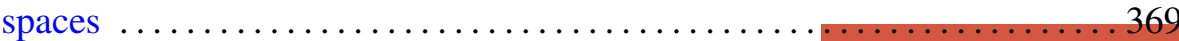

John R. Myers, Homology 3-spheres which admit no PL involutions . . . . . 379

Boon-Hua Ong, Invariant subspace lattices for a class of operators . . . . . 385

Chull Park, Representations of Gaussian processes by Wiener processes . . . 407

Lesley Millman Sibner and Robert Jules Sibner, A sub-elliptic estimate

for a class of invariantly defined elliptic systems $\ldots \ldots \ldots \ldots \ldots \ldots .417$

Justin R. Smith, Complements of codimension-two submanifolds. III.

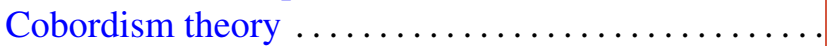

William Albert Roderick Weiss, Small Dowker spaces

David J. Winter, Cartan subalgebras of a Lie algebra and its ideals. II ... 